CARDIOVASCULAR MEDICINE

\title{
Transcoronary ablation of septal hypertrophy for hypertrophic obstructive cardiomyopathy: feasibility, clinical benefit, and short term results in elderly patients
}

\author{
F H Gietzen, C J Leuner, L Obergassel, C Strunk-Mueller, H Kuhn
}

Heart 2004;90:638-644. doi: 10.1136/hrt.2003.017509

See end of article for authors' affiliations

Correspondence to:

Dr F H Gietzen

Department of 'Cardiology,

Centre of Cardiovascular

Medicine, Salzburger Leite

1, D-97616 Bad

Neustadt/Saale,

Germany;

gietzen@t-online.de

Accepted

25 September 2003
Objective: To evaluate symptomatic and haemodynamic results of transcoronary ablation of septal hypertrophy for hypertrophic obstructive cardiomyopathy in elderly patients.

Setting: Tertiary referral centre for patients with hypertrophic obstructive cardiomyopathy.

Design: Retrospective study of two groups of consecutive patients divided at a median age (59 years).

Patients: Transcoronary ablation of septal hypertrophy was compared for 80 patients (group 1) $<60$ years of age and 77 patients (group 2) $\geqslant 60$ years of age. At baseline both groups were similar concerning the proportion of familial hypertrophic cardiomyopathy, concomitant moderate hypertension, prior syncope, left ventricular oufflow obstruction, left ventricular end diastolic pressure, and left ventricular ejection fraction. Patients in group 2 had a lower interventricular septal thickness and more severe disease as measured by New York Heart Association (NYHA) functional class, exercise capacity, pulmonary artery mean pressure at workload, and cardiac index at peak exercise.

Results: Median follow up was seven months after transcoronary ablation of septal hypertrophy. Both groups had a significant and similar improvement in basal and provokable obstruction, septal thickness, NYHA functional class, exercise tolerance, peak oxygen consumption, and pulmonary artery mean pressure at workload. Significant differences, compared with the younger group, were a higher proportion of persistent total atrioventricular block $(5 \% v 17 \%, p=0.015)$ and a slight decrease in left ventricular ejection fraction $(3(12) v-6(11) \%, p=0.001)$ in the elderly, despite a trend to a lower induced peak creatine kinase activity (596 (339) v 491 (331) U/I, $p=0.051$ ).

Conclusions: Short term results with transcoronary ablation of septal hypertrophy suggest that independent of a patient's age similar treatment strategies are justified in hypertrophic obstructive cardiomyopathy.
$\mathrm{P}$ atients with hypertrophic cardiomyopathy and an outflow tract gradient who have severe symptoms of heart failure unresponsive to medical treatment are candidates for intervention. ${ }^{1-4}$ The treatment most widely applied to such patients is surgical myectomy by removing a small amount of muscle from the basal interventricular septum. ${ }^{5-8}$ Surgery substantially reduces the subaortic outflow gradient in more than $90 \%$ of patients and results in persistent symptomatic improvement in about 70-90\%. ${ }^{12} 46-8$

In recent years, transcoronary ablation of septal hypertrophy by selective transcatheter septal branch injection of ethanol has been shown to reduce substantially outflow obstruction in $80-90 \%$ and symptoms in $84-90 \%$ of patients. ${ }^{9-17}$ Therefore, transcoronary ablation of septal hypertrophy may be an effective alternative to surgery. ${ }^{18-25}$ However, it remains a point of debate whether treatment of the elderly should be the same as for hypertrophic obstructive cardiomyopathy in young and middle aged patients. ${ }^{326-31}$ "Benign" processes associated with aging may contribute to the development of hypertrophic cardiomyopathy in the elderly, as opposed to more "malignant" processes that may be responsible for the development of idiopathic hypertrophy in younger patients. On the other hand, within the elderly group, there are those with severe disease who appear to have an adverse prognosis. ${ }^{29}$ Therefore, in older patients with severe symptoms a catheter intervention may be promising, since many of them may not be suitable candidates for surgery. ${ }^{11}$

The purpose of the present study was to compare the feasibility, clinical benefit, and short term results of transcoronary ablation of septal hypertrophy in two groups of consecutive patients: patients with hypertrophic obstructive cardiomyopathy, drug refractory severe symptoms, and $<60$ years of age (group 1); and patients with a similar clinical profile but $\geqslant 60$ years of age (group 2 ).

\section{METHODS \\ Patients}

The study comprised 157 consecutive patients ( 76 men) with hypertrophic obstructive cardiomyopathy who were treated by transcoronary ablation of septal hypertrophy at our institution between October 1995 and November 1999. The average (SD) age was 56 (16) years. None of the patients had significant coronary artery disease at the time of intervention. All patients had hypertrophic cardiomyopathy with subaortic obstruction based on typical clinical, echocardiographic, and angiocardiographic findings. Entry criteria included severe symptoms during daily activity, asymmetrical septal hypertrophy $\geqslant 15 \mathrm{~mm}$, systolic anterior motion of the mitral valve, and an intraventricular pressure gradient $\geqslant 30 \mathrm{~mm} \mathrm{Hg}$ in basal conditions or after provocation by a single premature ventricular beat ( $\geqslant 50 \mathrm{~mm} \mathrm{Hg}$ after two premature beats). Despite maximally tolerated doses of medical treatment with verapamil or $\beta$ blockers in all patients and additional diuretics in 35 patients (at the time of hospitalisation and at least three months before), 12 patients were in New York Heart Association (NYHA) functional class IV, 117 in class III,

Abbreviations: CK, creatine kinase; NYHA, New York Heart Association; PTCA, percutaneous transluminal coronary angioplasty 
and 28 in class II (treated for severe chest pain, symptomatic arrhythmias, syncope, or severe fatigue during daily activities). Permanent atrioventricular sequential pacing had failed to reduce symptoms in 14 patients. Syncope had occurred in 48 patients (31\%) and ventricular fibrillation in two. Concomitant systemic hypertension (> 160/95 mm Hg despite medical treatment for hypertrophic obstructive cardiomyopathy) was known in 11 patients. The mean (SD) septal thickness was 22 (4) $\mathrm{mm}$. The invasively measured mean (SD) resting gradient was 55 (42) $\mathrm{mm} \mathrm{Hg}$, the mean post-extrasystolic gradient was 147 (50) $\mathrm{mm} \mathrm{Hg}$, and the mean left ventricular end diastolic pressure was 20 (6) $\mathrm{mm} \mathrm{Hg}$. Family members were not systematically investigated. However, genetic transmission of the disease was evident by virtue of the history or echocardiographic documentation in 37 patients.

According to median age at baseline (59 years) the 157 study patients were divided into two groups. Group 1 consisted of 80 patients younger than 60 years of age and group 2 consisted of 77 patients who were 60 years of age or older (table 1).

The institutional review committee approved the study. All patients gave written informed consent.

\section{Investigations}

Clinical evaluation of the severity of symptoms, transthoracic echocardiography, and left and exercise right heart catheterisation were performed before transcoronary ablation of septal hypertrophy and at a median follow up of seven months (range 6-51 months) after the procedure. Exercise Doppler echocardiography was substituted for the invasive strategy for re-evaluation of the left ventricular outflow tract gradient in the last 51 consecutive patients. Patients' self perception of overall improvement was evaluated with a questionnaire that categorises the subjective feeling of wellbeing as improved, unchanged, or deteriorated. ${ }^{11}$

Transthoracic echocardiography was performed on an HP Sonos 1500 ultrasound machine and recorded on S-VHS video to allow serial review and side by side comparison of the studies. Basal interventricular septum thickness was derived from an integrated analysis of $M$ mode and two dimensional echocardiograms. Measurements were made according to the recommendations of the American Society of Echocardiography. ${ }^{32}$ Two dimensional echocardiographic images were obtained in the parasternal long and short axis views and apical two and four chamber views. The magnitude of the peak instantaneous left ventricular outflow tract gradient under basal conditions and during exercise was estimated with continuous wave Doppler. ${ }^{33}$

Exercise right heart catheterisation was performed with a 5 French Swan-Ganz catheter and a calibrated Statham P23ID strain gauge manometer. The catheter remained in the pulmonary artery for continuous monitoring of pulmonary artery pressures and to obtain blood samples for the calculation of cardiac output by the direct Fick method. Oxygen uptake was measured with use of a Vmax system 29c (SensorMedics Germany GmbH). Supine bicycle exercise was performed from an initial workload of $25 \mathrm{~W}$ to a maximum capacity with stepwise increases by $25 \mathrm{~W}$ every three minutes.

Coronary angiography and biplane ventriculography $\left(30^{\circ}\right.$ right anterior oblique and left lateral projections) were performed by standard techniques. Quantitative calculation of the left ventricular ejection fraction was based on a single plane $30^{\circ}$ right anterior oblique view.

Transcoronary ablation of septal hypertrophy was based on an "over the wire" percutaneous transluminal coronary angioplasty (PTCA) technique and has been described in detail. ${ }^{11}{ }^{12}$ A PTCA guiding catheter (6 or 7 French), a guide wire $(0.014$ inch), and a PTCA balloon catheter (diameter 1.5-2.5 mm, length $20 \mathrm{~mm}$ ) were used for the catheterisation of a small septal branch supplying the area of obstruction. The target vessel was identified by a functional approach that relies on the ischaemia induced decrease in outflow obstruction immediately after balloon inflation $(300-800 \mathrm{kPa}$, also to prevent reflux of ethanol).

Table 1 Baseline patient characteristics

\begin{tabular}{|c|c|c|c|}
\hline & Age $<60$ years & Age $\geqslant 60$ years & p Value \\
\hline Number of patients & 80 & 77 & \\
\hline Age (years) & $44(11)(21-59)$ & $69(5)(61-83)$ & $<0.001$ \\
\hline Male sex & $49(61 \%)$ & $27(35 \%)$ & 0.001 \\
\hline $\mathrm{FHCM}$ & $22(28 \%)$ & $15(19 \%)$ & 0.237 \\
\hline Hypertension & $4(5 \%)$ & $7(9 \%)$ & 0.315 \\
\hline NYHA functional class & $2.7(0.5)$ & $3.1(0.4)$ & $<0.001$ \\
\hline IV & 2 & 10 & \\
\hline III & 55 & 62 & \\
\hline II & 23 & 5 & \\
\hline Syncope & $23(29 \%)$ & $25(32 \%)$ & 0.613 \\
\hline Pre-implanted DDD pacemaker & $7(9 \%)$ & $7(9 \%)$ & 0.960 \\
\hline IVS thickness (mm) & $23(4)(16-38)$ & $22(3)(15-34)$ & 0.010 \\
\hline Left heart catheterisation & 80 & 77 & \\
\hline \multicolumn{4}{|l|}{ LVOT gradient } \\
\hline Rest $(\mathrm{mm} \mathrm{Hg})$ & $52(37)(0-140)$ & $57(47)(0-158)$ & 0.815 \\
\hline Post-ES (mm Hg) & $144(44)(40-236)$ & $150(56)(30-250)$ & 0.413 \\
\hline LVEDP $(\mathrm{mm} \mathrm{Hg})$ & $20(6)(8-38)$ & $19(6)(8-35)$ & 0.292 \\
\hline LVEF & $0.72(0.07)(0.60-0.87)$ & $0.73(0.07)(0.60-0.90)$ & 0.318 \\
\hline Right heart catheterisation & 75 & 69 & \\
\hline Workload (W) & $91(30)(0-175)$ & $57(28)(0-125)$ & $<0.001$ \\
\hline PAP $(\mathrm{mm} \mathrm{Hg})$ & 40 (9) $(20-59)$ & $43(10)(20-69)$ & 0.025 \\
\hline$\dot{\mathrm{V}}_{2} \max (\mathrm{ml} / \mathrm{kg} / \mathrm{min})$ & $17.4(4.5)(9.5-28.1)$ & $12.3(4.6)(3.9-24.3)$ & $<0.001$ \\
\hline$\dot{\mathrm{V}} \mathrm{O}_{2} \max (\%$ predicted) & $52(12)(28-82)$ & 49 (17) (17-92) & 0.363 \\
\hline $\mathrm{Cl}\left(\mathrm{l} / \mathrm{m}^{2} / \mathrm{min}\right)$ & $6.4(1.3)(3.5-9.8)$ & $5.0(1.7)(1.6-9.7)$ & $<0.001$ \\
\hline \multicolumn{4}{|c|}{$\begin{array}{l}\text { *Data are mean (SD) (range) or numbers (\%). } \\
\text { CI, cardiac index at maximum workload; FHCM, familial hypertrophic cardiomyopathy; IVS, interventricular } \\
\text { septal; LVEDP, left ventricular end diastolic pressure; LVEF, left ventricular ejection fraction; LVOT, left ventricular } \\
\text { oufflow tract; NYHA, New York Heart Association; PAP, pulmonary artery mean pressure at pretreatment } \\
\text { workload; Post-ES, post-extrasystolic gradient; Rest, resting gradient; } \mathrm{V}_{2} \text { max, oxygen consumption at maximum } \\
\text { workload. }\end{array}$} \\
\hline
\end{tabular}


The injection of radiographic contrast agent through the balloon catheter augments ischaemia and should be accompanied by a further decrease in outflow gradient if the target vessel has been correctly identified. Radiographic contrast agent also visualises the flow characteristics and the washout kinetics in the septal perforator artery. Furthermore, it marks the dimension of the myocardial area to be ablated. This pressure guided and angiography guided approach directs the application of ethanol (mean (SD) $3.2(2.1) \mathrm{ml}$ ) to a myocardial area that contributes to the outflow obstruction. Furthermore, it facilitates adaptation of the speed and amount of ethanol injected to the flow characteristics and the washout kinetics in the septal target vessel..$^{112125}$

Pressure was recorded continuously between a pigtail catheter at the apex of the left ventricle and the PTCA guiding catheter placed in the ostium of the left coronary artery. The outflow gradient (peak to peak) at rest and after provocation by stimulated ventricular extrasystoles (fixed coupling interval $370 \mathrm{~ms}$ ) were recorded. A right ventricular stimulation catheter was inserted to facilitate reproducible measurement of the post-extrasystolic gradient and to treat high grade atrioventricular block, which may develop during ethanol ablation of septal branches. ${ }^{11} 1425$

Post-procedural management included a right ventricular stimulation catheter and continuous ECG monitoring in the coronary care unit for up to four days. Creatine kinase (CK) activity was determined at hourly intervals during the first 24 hours. Heparin was administered by continuous intravenous infusion to reduce the risk of thrombus formation at the septal ablation site. Medical treatment with verapamil or $\beta$ blockers was continued during follow up for the initial 50 patients but was individualised (to treat supraventricular arrhythmias) afterwards. ${ }^{1125}$

\section{Statistical analysis}

Data processing and statistical analyses were performed with commercial software (SPSS release 6.0.1; SPSS Inc, Chicago, Illinois, USA) with the Pearson $\chi^{2}$ test for unordered categorical variables and the Mann-Whitney Wilcoxon test for ordered categorical variables. Student's $t$ test for paired and independent samples was used for normally distributed continuous variables and the Mann-Whitney Wilcoxon test was used for non-normally distributed continuous variables. A probability value of $\mathrm{p}<0.05$ (two tailed) was considered significant. Values are expressed as mean (SD).

\section{RESULTS}

\section{Baseline characteristics}

Compared with group 1 ( $<60$ years of age, mean (SD) 44 (11) years, $\mathrm{n}=80$ ), group 2 ( $\geqslant 60$ years of age, 69 (5) years, $\mathrm{n}=77$ ) had a significant female preponderance and patients had significantly lower basal interventricular septal thickness and a significantly higher NYHA functional class (table 1). Also, dyspnoea at rest occurred significantly more often in group 2 patients $(2.5 \% v 13 \%, \mathrm{p}=0.013)$, since 10 of 12 patients treated for NYHA functional class IV were $\geqslant 60$ years of age.

Exercise right heart catheterisation was performed in 75 of the patients in group 1 and in 69 of those in group 2. Group 2 patients had a significantly lower exercise capacity, maximum oxygen consumption, and cardiac index at peak exercise, as well as a significantly higher pulmonary artery mean pressure at workload. However, after adjustment for age and sex the maximum oxygen consumption as a percentage of the predicted value was not significantly different between the age groups. Group 1 and 2 patients were similar with respect to familial hypertrophic cardiomyopathy, concomitant moderate hypertension, and the incidence of prior syncope and DDD pacemaker implantation for hypertrophic obstructive cardiomyopathy. By left heart catheterisation both groups were similar with respect to resting and provokable outflow obstruction, left ventricular end diastolic pressure, and left ventricular ejection fraction.

\section{Transcoronary ablation of septal hypertrophy}

In group 2 patients the risk of a procedure related persistent total atrioventricular block and the proportion of pacemaker implantation were significantly higher, even though the peak CK activity values tended to be lower than in group 1 patients (table 2). Both groups were similar with respect to the amount of ethanol 96\% injected, the proportion of reinterventions (performed to eliminate provokable outflow obstruction completely as early as 8-12 days after a partially effective first procedure), ${ }^{11}$ and the number of septal vessels occluded in each patient.

\section{Complications}

Two patients in group 1 and two patients in group 2, all with known severe comorbidity, died during the first two weeks after transcoronary ablation of septal hypertrophy (fig 1). Therefore, the procedure related in-hospital mortality was similar and not significantly different between the groups $(2.5 \% \vee 2.6 \%, p=0.969)$. Causes of death were irreversible ventricular fibrillation $(\mathrm{n}=1)$ and progressive right heart failure $(n=1)$ in group 1 , and sudden total atrioventricular block $(\mathrm{n}=1)$ and penetration of a pacing lead with acute pericardial effusion $(n=1)$ in group 2 . However, three of these four patients (one from group 1 and two from group 2) belonged to a first series of patients who were treated with this new technique before September 1997. ${ }^{11} 21$

Other major complications (two in group 1 and three in group 2) were also similar and not significantly different between the groups $(2.5 \% \vee 3.9 \%, \mathrm{p}=0.618)$. In detail, the major complications were a severe but reversible spasm of the left coronary artery ${ }^{34}$ in two patients in group 1 , and an ischaemic stroke $(n=1)$, recurrent successfully terminated ventricular fibrillation $(\mathrm{n}=1)$, and a successful myectomy with mitral valve replacement after failure of transcoronary ablation of septal hypertrophy $(n=1)$ in group 2. All of these nine patients (four with in-hospital mortality and five with other major complications) were in NYHA functional class III $(\mathrm{n}=6)$ or IV $(\mathrm{n}=3)$ and presented with a resting gradient $\geqslant 30 \mathrm{~mm} \mathrm{Hg}$.

\section{Late death}

In group 1 a 31 year old woman had a witnessed instantaneous sudden death during an episode of severe emotional stress six months after intervention. Before transcoronary ablation of septal hypertrophy she had syncope, frequent ventricular extrasystoles, a subaortic septal thickness of $30 \mathrm{~mm}$, and a resting gradient of $72 \mathrm{~mm} \mathrm{Hg}$ (fig 1A). In group 2 five patients $(6.5 \%)$ died of non-cardiac causes (carcinoma in two patients, pulmonary embolism, liver cirrhosis, suicide), reflecting the high number of patients who were enrolled despite known severe comorbidity (fig 1B).

\section{Effect of transcoronary ablation of septal hypertrophy} Clinical evaluation

A median of seven months (mean (SD) 10 (8) months, range 6-51 months) after transcoronary ablation of septal hypertrophy clinical follow up was $100 \%$ complete for all patients remaining alive $(n=147)$, excluding one patient with myectomy $(\mathrm{n}=146)$. Clinical evaluation was obtained in 77 patients $<60$ years of age $(100 \%)$ and in 69 patients $\geqslant 60$ years of age $(100 \%)$. No significant differences were found between the two groups with respect to the magnitude of the 


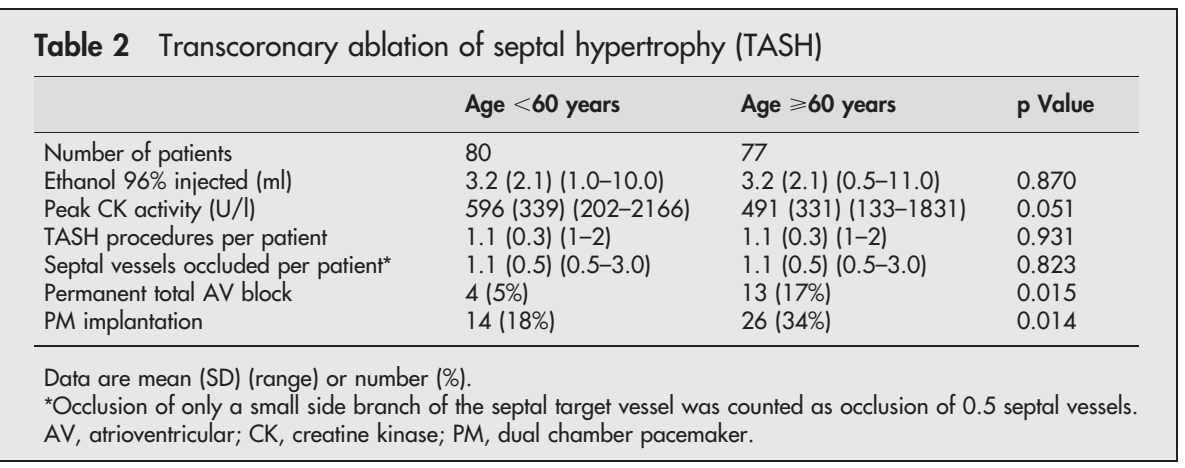

improvement in NYHA functional class and the reported overall subjective improvement of symptoms (table 3 ). In group 1 , all 77 patients $(100 \%)$ improved to class $\mathrm{I}(\mathrm{n}=51)$ or II $(n=26)$ (fig $1 \mathrm{~A})$. In group 2,61 patients $(88 \%)$ improved to class I $(\mathrm{n}=29)$ or II $(\mathrm{n}=32)$ and three patients $(4 \%)$ improved from class IV to class III. Five patients remained in class III (fig 1B).

\section{Haemodynamic function at rest}

A median of seven months (mean 7 (3) months, range 6-26 months) after transcoronary ablation of septal hypertrophy, haemodynamic function at rest $(88 \%$ complete follow up) was evaluated invasively $(\mathrm{n}=77)$ or by exercise Doppler echocardiography $(n=51)$ in 70 group 1 patients
(91\%) and in 58 group 2 patients (84\%). A significant decrease in left ventricular ejection fraction was found exclusively in patients who were 60 years of age or older, whereas group 1 patients had a small but not significant increase in left ventricular ejection fraction. The reduction in left ventricular ejection fraction was significantly correlated to patient age $(r=0.4, \mathrm{p}=0.001)$. No significant differences were found between the two groups with respect to the proportional reduction of the resting gradient, provokable gradient, and left ventricular end diastolic pressure. In groups 1 and 2 a similar and significant decrease of the resting gradient, provokable gradient, and left ventricular end diastolic pressure was found. By echocardiography the proportional reduction of the mean basal interventricular

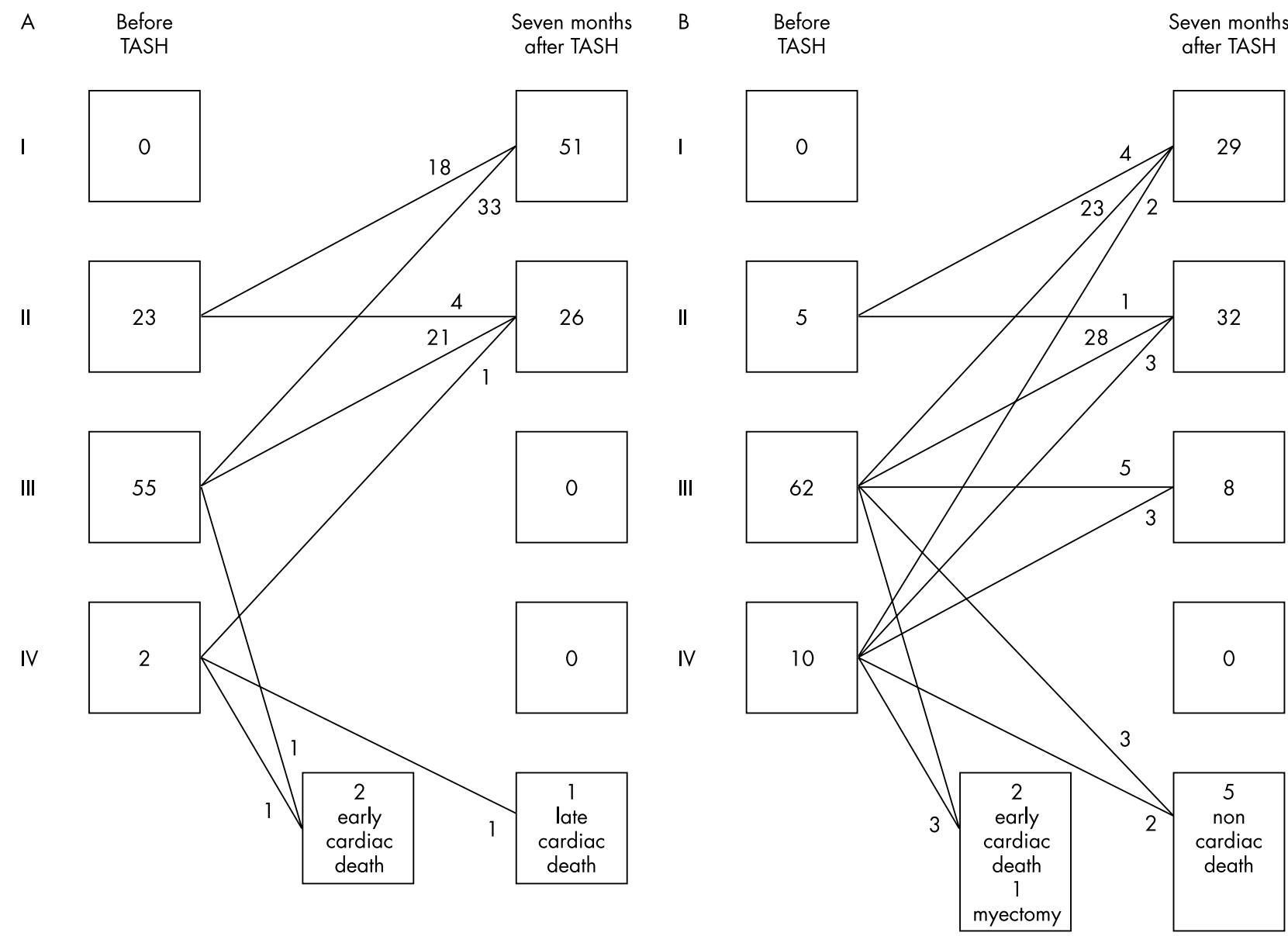

Figure 1 Changes in New York Heart Association (NYHA) functional class and mortality at a median follow up of seven months. NYHA functional class improved (A) from $2.7(0.4)$ to $1.4(0.5)(\mathrm{p}<0.001)$ in 80 patients $<60$ years of age (group 1) and (B) from $3.0(0.4)$ to $1.7(0.7)$ (p $<0.001)$ in 77 patients $\geqslant 60$ years of age (group 2). Cardiac and total mortality (in hospital and during follow up) were $3.8 \%$ and $3.8 \%$, respectively, of patients $<60$ years of age versus $2.6 \%$ and $9.1 \%$, respectively, of patients $\geqslant 60$ years of age. TASH, transcoronary ablation of septal hypertrophy. 


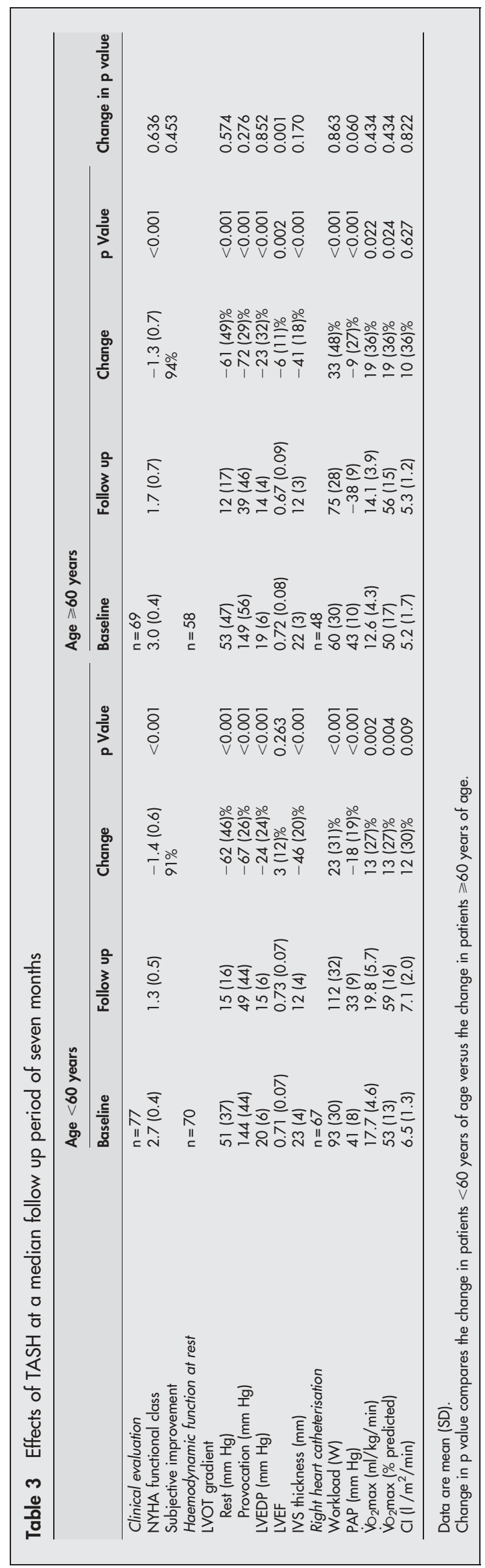

septal thickness was similar and significant in both groups (table 3).

\section{Exercise right heart catheterisation}

A median of seven months after transcoronary ablation of septal hypertrophy (mean (SD) 7 (3) months, range 6-26 months) exercise right heart catheterisation (79\% complete follow up) was performed in 67 group 1 patients (87\%) and in 48 group 2 patients (70\%). No significant differences were found between the groups with respect to the proportional improvement in exercise capacity, maximum oxygen consumption, pulmonary artery mean pressure at workload, and cardiac index at peak exercise (table 3 ). In group 1, as well as in group 2, significant improvements in exercise capacity, maximum oxygen consumption, and pulmonary artery mean pressure at workload were identified. Group 1 patients, in addition, had a significant increase in cardiac index at peak exercise. Patients with $(79 \%)$ and without $(21 \%)$ re-evaluation of exercise right heart catheterisation (because of severe comorbidity, age, or a lack of informed consent) had a similar and significant improvement in NYHA functional class $(-1.4$ (0.6) $v-1.3(0.7), \mathrm{p}=0.604)$.

\section{DISCUSSION}

Although it is often considered a disease of the young, hypertrophic obstructive cardiomyopathy is commonly diagnosed in the elderly. Studies have also indicated that this condition may be more common in older patients than is generally recognised, supporting the notion that the elderly may be a large and neglected subset of patients with hypertrophic obstructive cardiomyopathy. Whiting and colleagues ${ }^{35}$ reported that $32 \%$ of patients presenting with hypertrophic cardiomyopathy were $>60$ years of age, and in a community hospital based series published by Petrin and Tavel, $^{36} 83 \%$ of patients were $>50$ years of age. Krasnow and Stein $^{37}$ described 23 new cases of hypertrophic cardiomyopathy with 20 of the patients over the age of 50 years and 15 of these patients over the age of 60 years. Likewise, in a study by Lever and colleagues ${ }^{27}$ hypertrophic cardiomyopathy was more commonly diagnosed in patients $\geqslant 65$ years of age than in those $<40$ years of age. Nevertheless, most information about the pathophysiology, natural history, and treatment options of hypertrophic cardiomyopathy are derived from young and middle aged patients. Whether the disease takes the same form in the elderly, has the same pathophysiology, has the same genetic basis, or carries the same prognosis has not been clarified. ${ }^{26} 273839$

Fay and colleagues ${ }^{29}$ reported that elderly patients presenting with NYHA functional class I or II had a survival rate not different from that of control subjects and only $18 \%$ progressed to NYHA functional class III or IV during a 4.2 year follow up. Elderly patients presenting with NYHA functional class III or IV had a one year mortality of 36\%, which was significantly higher than that of control subjects. ${ }^{29}$ Lewis and Maron $^{28}$ followed up the clinical course of 52 severely symptomatic elderly patients ( 50 of 52 patients in NYHA functional class III or IV) with hypertrophic cardiomyopathy and found a high incidence of symptom progression and need for surgical myectomy.

In view of this, within the elderly group there are those with severe disease, categorised on the basis of symptoms and echocardiographic features of increased ventricular septal thickness and left atrial size, who appear to have an adverse prognosis. ${ }^{29}$ Particularly in older patients with severe symptoms a catheter intervention may be promising, since many of them may not be suitable candidates for surgery. ${ }^{11}$

According to median age at baseline ( 59 years), the present study compares the effects of transcoronary ablation of septal hypertrophy in consecutive patients with hypertrophic 
obstructive cardiomyopathy, drug refractory severe symptoms and $<60$ years of age, and in patients with a similar clinical profile but $\geqslant 60$ years of age.

Even in our group of patients $\geqslant 60$ years of age the baseline characteristics argued in favour of an idiopathic myocardial disease such as hypertrophic obstructive cardiomyopathy. Both age groups were similar and not significantly different concerning the proportion of familial hypertrophic cardiomyopathy, the prevalence of concomitant moderate hypertension, the incidence of prior syncope and the invasive measurements of left ventricular outflow obstruction (under basal and provokable conditions), left ventricular end diastolic pressure, and left ventricular ejection fraction. Since the lower exercise capacity in the elderly can be easily explained by their age and comorbidity, the remarkable and significant differences between the younger and the older patients were more severe dyspnoea at exertion, higher pulmonary artery mean pressure at workload, and significantly lower cardiac index at peak exercise in patients $\geqslant 60$ years of age. Even dyspnoea at rest was more frequently observed in the elderly, since 10 out of 12 patients treated for NYHA functional class IV were $\geqslant 60$ years of age.

Despite the argument that hypertrophic cardiomyopathy may develop in various age groups as a consequence of different pathological processes, ${ }^{29}$ our results support the use of similar treatment strategies in patients $<60$ years of age and in patients $\geqslant 60$ years of age. Transcoronary ablation of septal hypertrophy was equally effective in the elderly with respect to the reduction in outflow obstruction, subaortic septal thickness, and left ventricular end diastolic pressure. An almost identical proportion of patients $<60$ (91\%) and $\geqslant 60$ years of age $(94 \%)$ reported a subjective improvement. With respect to the improvement in NYHA functional class, exercise capacity, maximum oxygen consumption, pulmonary artery mean pressure at workload, and cardiac index at peak exercise, no significant differences were observed between the age groups.

The symptomatic and haemodynamic benefits in the elderly were accompanied by a slight but significant decrease in left ventricular ejection fraction that was not observed in the younger patients. Hypercontractility, characterised by an enhanced posterior excursion of the septum ${ }^{28}$ and more vigorous contractions remote from the area of ablation, persists to some degree in patients $<60$ years of age, despite an otherwise successful transcoronary ablation of septal hypertrophy and a tendency to higher induced peak CK activity. A further difference between the younger and the older patients was a significantly higher proportion of persistent total atrioventricular block $(5 \% \vee 17 \%)$ in the group of patients $\geqslant 60$ years of age. This may be explained by a higher intrinsic risk of total atrioventricular block in the elderly, since the ablation area tended to be smaller as estimated by a trend to a lower induced peak CK activity in the elderly. ${ }^{12}$ With regard to technical aspects (amount of ethanol injected, number of septal vessels occluded, and reinterventions performed), the catheter intervention appeared to be identical in both age groups.

Unlike surgical experience, in this study the procedure related early mortality and major complication rate were not influenced by patient age. In the elderly a lower basal interventricular septal thickness and a lower amount of ethanol injected may have counterbalanced the usually higher interventional risk of advanced age and may have influenced the problem of procedure related sustained ventricular arrhythmias during follow up. The arrhythmogenic risk of transcoronary ablation of septal hypertrophy depends on lesion size ${ }^{11}$ and may be reduced substantially in older patients with a lower burden of hypertrophy, who can be effectively treated by a lower induced peak CK activity. ${ }^{12} 40$
In elderly patients with symptomatic subaortic septal thickening, their disease may be related in part to hypertensive hypertrophy. ${ }^{26}$ However, there appears to be little evidence to support a consistent aetiological role for hypertension in the present study group. Only $9 \%$ of the elderly patients had evidence of moderate hypertension and blood pressure increases were well controlled by medical treatment. When the elderly group was subdivided into those with and those without hypertension, no significant differences existed between the groups concerning gradient reduction, relief of symptoms, and improvement of exercise haemodynamic variables. Furthermore, left ventricular outflow tract obstruction due to systolic anterior motion of the mitral valve, which was present in our study patients, is exceedingly uncommon in systemic hypertension. ${ }^{28}$ Nevertheless, it should be emphasised that our patients were a selected subgroup of severely symptomatic elderly patients with hypertrophic cardiomyopathy and are not representative of all patients $\geqslant 60$ years of age with this disease. Therefore, the results obtained should not be translated to patients with mild symptoms, effective medical treatment, or less objective substantiation of alterations in exercise haemodynamic variables.

\section{Conclusion}

Our present short term results with transcoronary ablation of septal hypertrophy suggest that, independent of patient age, similar treatment strategies are justified for severely symptomatic patients with hypertrophic obstructive cardiomyopathy.

\section{Authors' affiliations}

F H Gietzen, C J Leuner, L Obergassel, C Strunk-Mueller, H Kuhn, Department of Cardiology and Internal Intensive Care, Bielefeld Clinicum, Academic Teaching Hospital of the University of Muenster, Bielefeld, Germany

\section{REFERENCES}

1 Loogen F, Kuhn H, Gietzen F, et al. Clinical course and prognosis of patients with typical and atypical hypertrophic obstructive and with hypertrophic nonobstructive cardiomyopathy. Eur Heart J 1983;4(suppl F): 145-53.

2 Maron BJ, Bonow RO, Cannon RO, et al. Hypertrophic cardiomyopathy: interrelations of clinical manifestations, pathophysiology, and therapy. N Engl J Med 1987;316:780-9, 844-52.

3 Wigle ED, Rakowski H, Kimball BP, et al. Hypertrophic cardiomyopathy: clinical spectrum and treatment. Circulation 1995;92:1680-92.

4 Spirito $\mathbf{P}$, Seidman CE, McKenna WJ, et al. The management of hypertrophic cardiomyopathy. N Engl J Med 1997;336:775-85.

5 Morrow AG, Reitz BA, Epstein SE, et al. Operative treatment in hypertrophic subaortic stenosis: techniques, and the results of pre and postoperative assessments in 83 patients. Circulation 1975;52:88-102.

6 Maron BJ, Epstein SE, Morrow AG. Symptomatic status and prognosis of patients after operation for hypertrophic obstructive cardiomyopathy: efficacy of ventricular septal myotomy and myectomy. Eur Heart J 1983;4(suppl F): 175-85.

7 Schulte HD, Borisov K, Gams E, et al. Management of symptomatic hypertrophic obstructive cardiomyopathy: long-term results after surgical therapy. Thorac Cardiovasc Surg 1999;47:213-8.

8 Robbins RC, Stinson EB. Long-term results of left ventricular myotomy and myectomy for obstructive hypertrophic cardiomyopathy. J Thorac Cardiovasc Surg 1996;111:586-94.

9 Kuhn H, Gietzen F, Leuner Ch, et al. Induction of subaortic septal ischaemia to reduce obstruction in hypertrophic obstructive cardiomyopathy: studies to develop a new catheter-based concept of treatment. Eur Heart $J$ 1997; 18:846-51.

10 Sigwart U. Non-surgical myocardial reduction for hypertrophic obstructive cardiomyopathy. Lancet 1995;346:211-4.

11 Gietzen FH, Leuner ChJ, Raute-Kreinsen U, et al. Acute and long-term results after transcoronary ablation of septal hypertrophy (TASH): catheter interventional treatment for hypertrophic obstructive cardiomyopathy. Eur Heart J 1999;20:1342-54

12 Kuhn H, Gietzen FH, Schäfers M, et al. Changes in the left ventricular outflow tract after transcoronary ablation of septal hypertrophy (TASH) for hypertrophic obstructive cardiomyopathy as assessed by transoesophageal echocardiography and by measuring myocardial glucose utilization and perfusion. Eur Heart J 1999;20:1808-17.

13 Knight Ch, Kurbaan AS, Seggewiss $\mathrm{H}$, et al. Nonsurgical septal reduction for hypertrophic obstructive cardiomyopathy: outcome in the first series of patients. Circulation 1997;95:2075-81. 
14 Faber L, Seggewiss H, Gleichmann U. Percutaneous transluminal septal myocardial ablation in hypertrophic obstructive cardiomyopathy: results with respect to intraprocedural myocardial contrast echocardiography. Circulation 1998:98:2415-21.

15 Lakkis NM, Nagueh SF, Kleimann NS, et al. Echocardiography-guided ethanol septal reduction for hypertrophic obstructive cardiomyopathy. Circulation 1998;98:1750-5.

16 Boekstegers P, Steinbigler P, Molnar A, et al. Pressure-guided nonsurgical myocardial reduction induced by small septal infarctions in hypertrophic obstructive cardiomyopathy. J Am Coll Cardiol 2001;38:846-53.

17 Mazur W, Nagueh SF, Lakkis NM, et al. Regression of left ventricular hypertrophy after nonsurgical septal reduction therapy for hypertrophic obstructive cardiomyopathy. Circulation 2001;103:1492-6.

18 Nagueh SF, Ommen SR, Lakkis NM, et al. Comparison of ethanol septal reduction therapy with surgical myectomy for the treatment of hypertrophic obstructive cardiomyopathy. J Am Coll Cardiol 2001;38:1701-6.

19 Wigle ED, Schwartz L, Woo A, et al. To ablate or operate? That is the Question! J Am Coll Cardiol 2001;38:1707-10.

20 Fananapazir L, McAreavey D. Therapeutic options in patients with obstructive hypertrophic cardiomyopathy and severe drug-refractory symptoms. J Am Coll Cardiol 1998;31:259-64.

21 Kuhn H, Gietzen FH, Leuner Ch, et al. Transcoronary ablation of septal hypertrophy (TASH): a new treatment option for hypertrophic obstructive cardiomyopathy. Z Kardiol. 2000;89: IV/41-54.

22 Spirito P, Maron BJ. Perspectives on the role of new treatment strategies in hypertrophic obstructive cardiomyopathy. J Am Coll Cardiol 1999;33:1071-5.

23 Maron BJ. New interventions for obstructive hypertrophic cardiomyopathy: promise and prudence. Eur Heart J 1999;20:1292-4.

24 Braunwald $\mathbf{E}$. Hypertrophic cardiomyopathy: the benefits of a multidisciplinary approach. N Engl J Med 2002;347:1306-7.

25 Gietzen FH, Leuner ChJ, Hegselmann J, et al. [letter]. Eur Heart J 2000;21:590-5

26 Topol EJ, Traill TA, Fortuin NJ. Hypertensive hypertrophic cardiomyopathy of the elderly. N Engl J Med 1985;312:277-83.

27 Lever HM, Karam RF, Currie PJ, et al. Hypertrophic cardiomyopathy in the elderly: distinctions from the young based on cardiac shape. Circulation 1989;79:580-9.
28 Lewis JF, Maron BJ. Elderly patients with hypertrophic cardiomyopathy: a subset with distinctive left ventricular morphology and progressive clinical course late in life. J Am Coll Cardiol 1989;13:36-45.

29 Fay WP, Taliercio CP, Ilstrup DM, et al. Natural history of hypertrophic cardiomyopathy in the elderly. J Am Coll Cardiol 1990;16:821-6.

30 Chikamori T, Doi YL, Yonezawa Y, et al. Comparison of clinical features in patients $\geqslant 60$ years of age and those $\leqslant 40$ years of age with hypertrophic cardiomyopathy. Am J Cardiol 1990;66:875-8.

31 Maron BJ, Olivotto I, Spirito P, et al. Epidemiology of hypertrophic cardiomyopathy-related death revisited in a large non-referral-based patient population. Circulation 2000;102:858-64.

32 Sahn DJ, De Maria A, Kisslo J, et al. Recommendations regarding quantitation in M-mode echocardiography: results of a survey of echocardiographic measurements. Circulation 1978;58:1072-83.

33 Panza JA, Petrone RK, Fananapazir L, et al. Utility of continuous wave Doppler echocardiography in noninvasive assessment of the left ventricular oufflow tract pressure gradient in patients with hypertrophic cardiomyopathy. J Am Coll Cardiol 1992;19:91-9.

34 Kuhn H, Gietzen F, Leuner C. "The abrupt no-flow": a no-reflow like phenomenon in hypertrophic cardiomyopathy [letter]. Eur Heart J 2002;23:91-3.

35 Whiting RB, Powell WJ, Dinsmore RE, et al. Idiopathic hypertrophic subaortic stenosis in the elderly. N Engl J Med 1971;285:196-200.

36 Petrin TJ, Tavel ME. Idiopathic hypertrophic subaortic stenosis as observed in a large community hospital: relation to age and history of hypertension. J Am Geriatr Soc 1979;27:43-6

37 Krasnow N, Stein RA. Hypertrophic cardiomyopathy in the aged. Am Heart J 1978;96:326-36

38 Zieman SJ, Fortuin NJ. Hypertrophic and restrictive cardiomyopathies in the elderly. Cardiol Clin 1999;17:159-72.

39 Kitzman DW, Scholz DG, Hagen PT, et al. Age-related changes in normal human hearts during the first 10 decades of life. Part II (maturity). A quantitative anatomic study of 765 specimens from subjects 20 to 99 years old. Mayo Clin Proc 1988;63:137-46.

40 Wigle ED, Sasson Z, Henderson MA, et al. Hypertrophic cardiomyopathy: the importance of the site and the extent of hypertrophy: a review. Prog Cardiovasc Dis 1985;28:1-83.

\section{FROM BMJ JOURNALS \\ Role of "Ischemia Modified Albumin", a new biochemical marker of myocardial ischaemia, in the early diagnosis of acute coronary syndromes \\ M K Sinha, D Roy, D C Gaze, P O Collinson, J-C Kaski}

Pease visit the Heart website [www.heartinl. com] for a link to the full text of this article.
Background: Diagnosis of cardiac ischaemia in patients attending emergency departments (ED) with symptoms of acute coronary syndromes is often difficult. Cardiac troponin (cTn) is sensitive and specific for the detection of myocardial damage but may not rise during reversible myocardial ischaemia. Ischemia Modified Albumin (IMA) has recently been shown to be a sensitive and early biochemical marker of ischaemia.

Methods and Results: This study evaluated IMA in conjunction with ECG and cTn in 208 patients presenting to the ED within three hours of acute chest pain. At presentation, a 12lead ECG was recorded and blood taken for IMA and cardiac troponin T (cTnT). Patients underwent standardised triage, diagnostic procedures, and treatment. Results of IMA, ECG, and CTnT, alone and in combination, were correlated with final diagnoses of non-ischaemic chest pain, unstable angina, ST segment elevation, and non-ST segment elevation myocardial infarction. In the whole patient group, sensitivity of IMA at presentation for an ischaemic origin of chest pain was $82 \%$, compared with $45 \%$ of ECG and $20 \%$ of cTnT. IMA used together with CTnT or ECG, had a sensitivity of $90 \%$ and $92 \%$, respectively. All three tests combined identified $95 \%$ of patients whose chest pain was attributable to ischaemic heart disease. In patients with unstable angina, sensitivity of IMA used alone was equivalent to that of IMA and ECG combined.

Conclusions: IMA is highly sensitive for the diagnosis of myocardial ischaemia in patients presenting with symptoms of acute chest pain.

A Emergency Medicine Journal 2004;21:29-34. 